\title{
Trace anomaly contribution to hydrogen atom mass
}

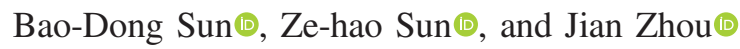 \\ Key Laboratory of Particle Physics and Particle Irradiation (MOE), Institute of Frontier \\ and Interdisciplinary Science, Shandong University, (QingDao), Shandong 266237, China
}

(Received 3 January 2021; accepted 19 August 2021; published 9 September 2021)

\begin{abstract}
We compute trace anomaly contribution to hydrogen atom mass, which turns out to be related to the part of the Lamb shift. To the best of our knowledge, this is a first model independent calculation of trace anomaly contribution to a bound state. This finding might shed new light on our understandings of the mass structure of QCD bound states, such as, heavy quarkonium.
\end{abstract}

DOI: $10.1103 /$ PhysRevD.104.056008

\section{INTRODUCTION}

The origin of proton mass is one of the most fundamental questions remain to be answered in hadronic physics study. The current light quark masses generated through the BroutEnglert-Higgs mechanism only makes up a small part of proton mass. At the classical level, the proton mass vanishes identically in the massless limit due to the exact cancellation between the quark's kinetic energy and the negative potential energy according to the relativistic virial theorem [1], the field theory formulation of which states that the trace of the QCD energy momentum tensor (EMT) vanishes in the chiral limit. Therefore, the large piece of proton mass essentially originates from the quantum effect, i.e., the trace anomaly of the energy momentum tensor [2-4], which comes as the consequence of the violation of the approximate conformal symmetry of QCD [4].

Due to the nonperturbative nature of low energy QCD, it appears to be impossible to solve the problem analytically. Instead, one can try to gain some insights into the proton mass structure by decomposing it into different pieces $[5,6]$ based on the QCD EMT, namely, trace anomaly contribution, parton kinematic energy and potential energy. These different contributions can be computed in lattice QCD or measured in high energy scatterings [7-13]. In particular, the tremendous progress on lattice calculations has been made in recent years [14-18]. On the other hand, the proton mass decomposition issue [19-22] and the renormalization properties of the different terms of the energy momentum tensor [9,10,22,23], have gained renewed interest previously. We recognized that the QCD EMT also plays a key role in the various different context of strongly interacting matter studies [24-29].

Published by the American Physical Society under the terms of the Creative Commons Attribution 4.0 International license. Further distribution of this work must maintain attribution to the author(s) and the published article's title, journal citation, and DOI. Funded by SCOAP .
As mentioned above, most previous studies of trace anomaly focus on its nonperturbative origin and formal aspects, such as its contribution to proton mass and renormalization properties. In this short note, we make an attempt to promote our understanding of the trace anomaly contribution to bound state mass with a different strategy. Namely, we compute the contribution from the trace anomaly of the QED EMT to the mass of the simplest QED bound state, i.e., hydrogen atom, and relate it to other known physical quantities since this is a completely solvable problem in quantum mechanics. To be more specific, trace anomaly contribution is related to part of the Lamb shift. This is in sharp contrast to proton mass decomposition case $[5,6]$ where trace anomaly part is considered to be a new additional contribution to the bound state mass other than parton kinematic energy and potential energy. To the best of our knowledge, this is the first field theory calculation for trace anomaly contribution to a real world bound state. It is the first step toward the understanding of trace anomaly contribution to bound state mass in a perturbation theory, and thus is interesting in its own right. The method developed in this note can potentially be applied to analysis the mass decomposition of the heavy quarkonium, for which case, the perturbative treatment is justified to some extend.

The note is structured as follows. In the next section, we present the detailed derivation of trace anomaly contribution to hydrogen atom mass and show its relation with the Lamb shift. In Sec. III, we summarize our work and discuss the possible extension of the calculation to heavy quarkonium case.

\section{TRACE ANOMALY CONTRIBUTION TO HYDROGEN ATOM MASS}

We begin with introducing the trace of the QED EMT,

$$
T_{\mu}^{\mu}=\left(1+\gamma_{m}\right) m_{0} \bar{\Psi} \Psi+\frac{\beta(e)}{2 e}\left[F^{\mu \nu} F_{\mu \nu}\right]_{R},
$$


where $\gamma_{m}=\frac{3 \alpha}{2 \pi}$ and $\frac{\beta(e)}{2 e}=\frac{\alpha}{6 \pi}$ at the next to leading order. The subscript $R$ indicates that the gauge field is the renormalized one $[2,30] . m_{0}$ is electron bare mass. Notice that the first operator is renormalization invariant [31], so that one has $m_{0} \bar{\Psi} \Psi=[m \bar{\Psi} \Psi]_{R}+O(\alpha)$.

The main purpose of this paper is to investigate the trace anomaly contribution to the hydrogen atom mass from the operator $\frac{\beta(e)}{2 e}\left[F^{\mu \nu} F_{\mu \nu}\right]_{R}$ and relate it to the known physical quantity. It is also interesting to compute the leading order correction and the next to leading order correction to the operator $\left(1+\gamma_{m}\right) m_{0} \bar{\Psi} \Psi$, not only from the point of view of making our calculation self-contained, but also for deepen our understanding of the role played by the mass term in a perturbation theory, which might be relevant for the study of mass decomposition of heavy quarkonium. We found that the combination of the trace anomaly contribution and the NLO correction to the $m_{0} \bar{\Psi} \Psi$ from the vacuum polarization diagram is related to the Uehling effect which is the small part of the Lamb shift. The difference between the NLO corrections to the operator $\left(1+\gamma_{m}\right) m_{0} \bar{\Psi} \Psi$ in the vacuum and in the bound state gives rise to the rest part of the Lamb shift. Our calculation thus provides a nice consistency check for the mass decomposition formula presented in Eq. (1) in a perturbation theory.

We first compute the leading order contribution to the expectation value of the operator $m_{0} \bar{\Psi} \Psi$ for the ground state of hydrogen atom. For simplicity, the proton inside hydrogen atom merely serves as an infinitely heavy charge source and thus becomes decoupled in our treatment. From Poincare invariance, the matrix elements of the QED EMT in a hydrogen atom state with momentum $P$ reads,

$$
\left\langle P\left|T^{\mu \nu}\right| P\right\rangle=2 P^{\mu} P^{\nu}
$$

where the state is normalized according to

$$
\left\langle P^{\prime} \mid P\right\rangle=2 P^{0}(2 \pi)^{3} \delta^{3}\left(P^{\prime}-P\right) .
$$

The hydrogen atom mass can be related to the trace of the QED EMT,

$$
\left\langle P\left|T_{\mu}^{\mu}\right| P\right\rangle=2 M^{2} .
$$

Let us now work in the rest frame. By noticing that $\langle P \mid P\rangle=2 M \int d^{3} x$, the hydrogen atom mass at the leading order is given by(omitting proton mass),

$$
\begin{aligned}
M_{H, 0} & =\frac{\left\langle H\left|\int d^{3} x m_{0} \bar{\Psi}(x) \Psi(x)\right| H\right\rangle}{\langle H \mid H\rangle} \\
& =m \int d^{3} x \varphi_{0}^{\dagger}(x) \gamma^{0} \varphi_{0}(x)
\end{aligned}
$$

where $m_{0}=m$ at the leading order with $m$ being the electron physical mass. This relation was first discovered by V. Fock [32]. $\varphi_{0}(x)$ is the ground state wave function normalized to $\int \varphi_{0}^{\dagger}(x) \varphi_{0}(x) d^{3} x=1$. Here we changed the notation from $\langle P|$ to $\langle H|$. Plugging the wave function of the ground state computed from the Dirac equation, we derive,

$$
M_{H, 0}=m \sqrt{1-\alpha^{2}} .
$$

The difference between $M_{H, 0}$ and electron mass is given by,

$$
M_{H, 0}-m=m \sqrt{1-\alpha^{2}}-m \approx-13.6 \mathrm{eV}
$$

which is precisely the ground state energy of hydrogen atom. Therefore, the EM potential energy and electron kinematic energy is implicitly included in the electron mass term.

We now proceed to compute the trace anomaly contribution and the NLO correction to the electron mass term. At higher order, two operators $m_{0} \bar{\Psi}(x) \Psi(x)$ and $F^{\mu \nu}(x) F_{\mu \nu}(x)$ mix with each other under renormalization. As pointed out in Refs. [22,23], the trace anomaly contribution to the mass of the free electron state is scheme dependent, and perhaps is not a physical observable. What matters is the difference between the anomaly contribution to the mass of free particle and that in a bound state. Here we specify the subtraction scheme following Refs. [2,22,23],

$$
\begin{aligned}
& \left\langle e\left|\left[F^{\mu \nu}(x) F_{\mu \nu}(x)\right]_{R}\right| e\right\rangle=0 \\
& \left\langle\gamma\left|\left[F^{\mu \nu}(x) F_{\mu \nu}(x)\right]_{R}\right| \gamma\right\rangle=\left\langle\gamma\left|Z_{3}^{-1} F^{\mu \nu}(x) F_{\mu \nu}(x)\right| \gamma\right\rangle_{\text {Tree }}
\end{aligned}
$$

which appears to be the most natural choice. By inserting the time evolution operator, we calculate the expectation value of the trace anomaly part with,

Fig. $1(a)=\frac{\left\langle H\left|\int d^{3} x \frac{\beta}{2 e}\left[F^{\mu \nu}(x) F_{\mu \nu}(x)\right]_{R} \mathcal{T} e^{-i \int d^{4} y H_{I}(y)}\right| H\right\rangle}{\langle H \mid H\rangle}$.

The Feynman diagram contributing to this matrix element at the first nontrivial order is shown in Fig. 1(a). By applying the standard Feynman rules in the Coulomb gauge in which the Coulomb photon propagator simply takes the form $\frac{-i}{\vec{k}^{2}}[33,34]$, we have the relation,

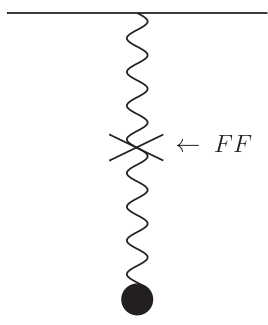

(a)

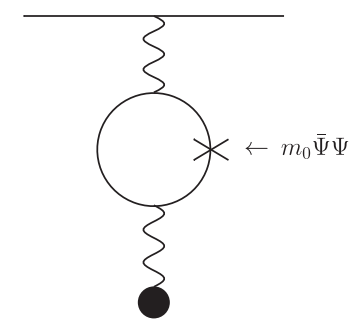

(b)
FIG. 1. Trace anomaly contribution (diagram a) and the vacuum polarization diagram with the mass operator insertion (diagram b). Black dots represent the interaction with Coulomb potential. 
Fig. $1(a)=-\frac{4}{3} \alpha^{2} \int d^{3} y \int \frac{d^{3} q}{(2 \pi)^{3}} \frac{e^{i \vec{q} \cdot \vec{y}}}{\vec{q}^{2}+i \epsilon}\left[\bar{\varphi}_{0}(y) \gamma^{0} \varphi_{0}(y)\right]$. which is just the trace anomaly contribution to hydrogen atom mass. At the NLO, the expectation value of the electron mass term receives the contribution from the vacuum polarization diagram Fig. 1(b) [35],

$$
2 \times \text { Fig. } 1(b)=8 \alpha^{2} \int d^{3} y \int \frac{d^{3} q}{(2 \pi)^{3}} \frac{e^{i \vec{q} \cdot \vec{y}}}{\vec{q}^{2}+i \epsilon} \int_{0}^{1} d a \frac{a(1-a) m^{2}}{m^{2}+a(1-a) \vec{q}^{2}}\left[\bar{\varphi}_{0}(y) \gamma^{0} \varphi_{0}(y)\right]
$$

It is convenient to group these two contributions together,

$$
\text { Fig. } 1(a)+2 \times \text { Fig. } 1(b) \approx-8 \alpha^{2} \int d^{3} y \int \frac{d^{3} q}{(2 \pi)^{3}} e^{i \vec{q} \cdot \vec{y}} \int_{0}^{1} d a \frac{a^{2}(1-a)^{2}}{m^{2}} \varphi_{0}^{\dagger}(y) \varphi_{0}(y)=\frac{-4 \alpha^{2}}{15 m^{2}} \varphi_{0}^{\dagger}(0) \varphi_{0}(0)
$$

To arrive at the above expression, we have made the Taylor expansion in terms of the power $\frac{\vec{q}^{2}}{m^{2}}$. This turns out to be just the part of the Lamb shift that is caused by the vacuum polarization effect.

We now turn to the calculation of the self-energy corrections. As a warm up exercise, we first compute the self-energy corrections to the mass of free electron state as shown in Figs. 2. It is straightforward to obtain,

$$
\begin{gathered}
\text { Fig. } 2(a)=\gamma_{m} m_{0}+m-\delta m=\gamma_{m} m_{0}+m-\frac{\alpha}{2 \pi} m_{0} \int_{0}^{1} d a(2-a) \ln \frac{a \Lambda^{2}}{(1-a)^{2} m_{0}^{2}} \\
\text { Fig. } 2(b)=\frac{\alpha}{2 \pi} m_{0} \int_{0}^{1} d a\left\{2 \ln \frac{a \Lambda^{2}}{(1-a)^{2} m_{0}^{2}}-\frac{2(2-a)}{(1-a)}\right\} \\
2 \times \text { Fig. } 2(c)=\frac{\alpha}{2 \pi} m_{0} \int_{0}^{1} d a\left\{-a \ln \frac{a \Lambda^{2}}{(1-a)^{2} m_{0}^{2}}+\frac{2 a(2-a)}{(1-a)}\right\}
\end{gathered}
$$

where $\Lambda$ is the UV regulator in the Pauli-Villars regularization. Summing up all three terms, one obtains,

$$
\frac{\left\langle e\left|\left(1+\gamma_{m}\right) m_{0} \int d^{3} x \bar{\Psi}(x) \Psi(x)\right| e\right\rangle}{\langle e \mid e\rangle}=m
$$

As expected, at the NLO, the physical mass of a free electron entirely comes from the electron mass term and the associated anomaly part. An all order proof of Eq. (16) in the subtraction scheme specified above can be achieved by invoking the Callan-Symanzik equation [2].
Now we move on to compute the difference of the self-energy correction between a free electron state and that in the bound state. The self-energy correction of electron in hydrogen atom still arises from the loop diagrams Fig. 2(b) and Fig. 2(c), but with the internal electron propagator being replaced by the one computed in the presence of the background Coulomb field. The calculation can be most conveniently formulated using the NRQED in the Coulomb gauge. The effective Lagrangian of NRQED is obtained from the full QED Lagrangian,

$$
\mathcal{L}=\psi^{\dagger}\left(i \partial^{0}-e A^{0}-\frac{\vec{p}^{2}}{2 m_{0}}+\frac{e}{2 m_{0}}\left(\vec{p}^{\prime}+\vec{p}\right) \cdot \vec{A}-\frac{e^{2}}{2 m_{0}} \vec{A}^{2}-(1+O(\alpha)) \frac{i e}{2 m_{0}} \sigma \cdot\left[\left(\vec{p}-\vec{p}^{\prime}\right) \times \vec{A}\right]\right) \psi+\cdots
$$

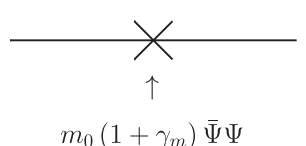

(a)

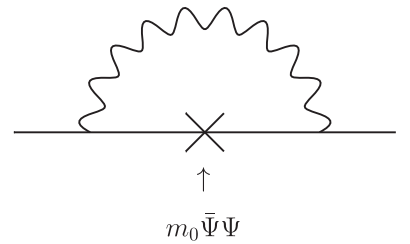

(b)

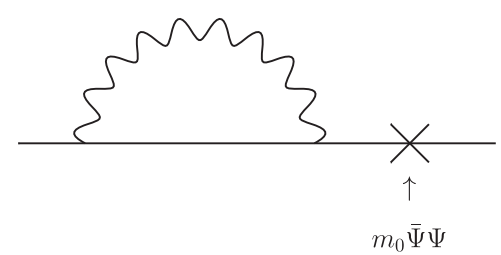

(c)

FIG. 2. NLO corrections to the electron mass term. 
by expressing the four component spinor $\Psi$ in terms of two component spinor $\psi$ in the nonrelativistic limit,

$$
\Psi \approx e^{-i m_{0} t} \frac{1}{\sqrt{2}}\left(\begin{array}{c}
\left(1-\frac{\vec{\sigma} \cdot(\vec{p}-e \vec{A})}{2 m_{0}}\right) \psi \\
\left(1+\frac{\vec{\sigma} \cdot(\vec{p}-e \vec{A})}{2 m_{0}}\right) \psi
\end{array}\right)
$$

where $\vec{p}$ and $\vec{p}^{\prime}$ are three momentum operators acting on the fields $\psi$ and $\psi^{\dagger}$ respectively.

The contribution from Fig. 2(c) can be expressed as $\delta Z_{2} m_{0} \bar{\Psi}(x) \gamma^{\mu} \Psi(x)$. Due to the conservation of the vector current in QED, the calculation of $\delta Z_{2} m_{0} \bar{\Psi}(x) \gamma^{\mu} \Psi(x)$ can be converted to compute $-\delta F_{1}(0) m_{0} \bar{\Psi}(x) \gamma^{\mu} \Psi(x)$ where $\delta F_{1}(0)$ denotes the first order correction to the vertex function $F(0)$ at zero momentum transfer,

$$
\left\langle H\left|\int d^{3} x \bar{\Psi}_{R}(x) \gamma^{\mu} \Psi_{R}(x)\right| H\right\rangle_{\mathrm{NLO}}=\delta F_{1}(0)\left\langle H\left|\int d^{3} x \bar{\Psi}_{R}(x) \gamma^{\mu} \Psi_{R}(x)\right| H\right\rangle_{\text {Tree }}
$$

where the subscript $\bar{\Psi}_{R}$ stands for the renormalized field. It is most convenient to choose the Lorentz index $\mu$ to be 0 . One then has,

$$
\begin{aligned}
2 \times \text { Fig. 2(c) } & =-\frac{\left\langle H\left|m_{0} \int d^{3} x \bar{\Psi}_{R}(x) \gamma_{0} \Psi_{R}(x)\right| H\right\rangle_{\mathrm{NLO}}}{\left\langle H\left|\int d^{3} x \Psi_{R}^{\dagger}(x) \Psi_{R}(x)\right| H\right\rangle_{\mathrm{Tree}}} \\
& =-\frac{\left\langle H\left|m_{0} \int d^{3} x \Psi_{R}^{\dagger}(x) \Psi_{R}(x)\right| H\right\rangle_{\mathrm{NLO}}}{\langle H \mid H\rangle}
\end{aligned}
$$

Combining the above with Fig. 2(b), one obtains,

$$
\text { Fig. } 2(b)+2 \times \text { Fig. } 2(c)=\frac{\left\langle H\left|m_{0} \int d^{3} x\left[\bar{\Psi}_{R}(x) \Psi_{R}(x)-\Psi_{R}^{\dagger}(x) \Psi_{R}(x)\right]\right| H\right\rangle_{\mathrm{NLO}}}{\langle H \mid H\rangle}
$$

which can be converted into the following form with the help of Eq. (18),

$$
\text { Fig. } 2(b)+2 \times \text { Fig. } 2(c) \approx \frac{\left\langle H\left|\int d^{3} x\left\{\psi^{\dagger}\left[\frac{e}{2 m_{0}}\left(\vec{p}^{\prime}+\vec{p}\right) \cdot \vec{A}-\frac{\vec{p}^{2}}{2 m_{0}}-\frac{e^{2}}{2 m_{0}} \vec{A}^{2}-\frac{i e}{2 m_{0}} \sigma \cdot\left[\left(\vec{p}-\vec{p}^{\prime}\right) \times \vec{A}\right]\right] \psi\right\}\right| H\right\rangle}{\langle H \mid H\rangle}
$$

where the operators $\psi^{\dagger}$ and $\psi$ should be understood as the renormalized ones. It is easy to verify that the spin-orbital coupling term $-\frac{i e}{2 m_{0}} \sigma \cdot\left[\left(\vec{p}-\vec{p}^{\prime}\right) \times \vec{A}\right]$ does not contribute to the mass shift. We are now ready to compute the expectation values of these operators in NRQED.

One first notices that the operators $\psi^{\dagger} \frac{\vec{p}^{2}}{2 m_{0}} \psi, \psi^{\dagger} \frac{e^{2}}{2 m_{0}} \vec{A}$. $\vec{A} \psi$ in a free electron state and in a bound state yield the same contribution at the order of interest [36]. The energy

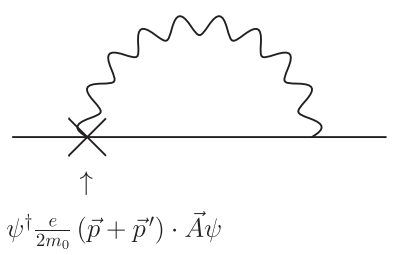

(a)

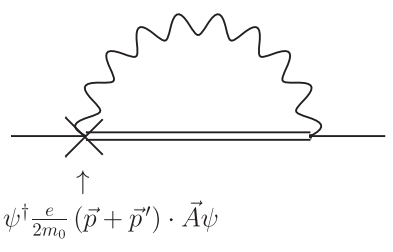

(b)
FIG. 3. Part of the NLO correction to the electron mass term in NRQED in vacuum (diagram a), and that in the bound state (diagram b). shift entirely comes from the operator $\psi^{\dagger} \frac{e}{2 m_{0}}\left(\vec{p}^{\prime}+\vec{p}\right) \cdot \vec{A} \psi$, the expectation value of which can be diagrammatically computed as shown in Fig. 3. Note that Fig. 3 represents the part of the NLO correction from Fig. 2 which can lead to the energy level shift. By applying the standard Feynman rules of NRQED in the Coulomb gauge for transverse photon propagator $\frac{-i}{\vec{k}^{2}}\left(\delta^{i j}-\frac{\vec{k}^{i} \vec{k}^{j}}{\vec{k}^{2}}\right)[33,34]$, the Fig. 3(a) reads,

$$
\begin{aligned}
& \text { Fig. } 3(a)=\frac{\alpha}{(2 \pi)^{2}}\left(\int d^{3} x \varphi_{0}^{\dagger}(x) \frac{\nabla^{2}}{m_{0}^{2}} \varphi_{0}(x)\right) \\
& \times \int d^{3} k\left[\frac{2}{3 \vec{k}^{2}}-\frac{1-\vec{k}^{2} /\left(3\left(\vec{k}^{2}+\mu^{2}\right)\right)}{\vec{k}^{2}+\mu^{2}}\right] .
\end{aligned}
$$

To arrive at the above expression, we have imposed a scale cut off $\mu$ by replacing the photon propagator $\frac{1}{k^{2}+i \epsilon} \rightarrow$ $\frac{1}{k^{2}+i \epsilon}-\frac{1}{k^{2}-\mu^{2}+i \epsilon}$ following the Weinberg's method [36]. In a bound state, the internal electron propagator in the 
position space is given by $\sum_{M} \frac{\varphi_{M}(x) \varphi_{M}^{\dagger}(y)}{\Delta E_{M}+i \epsilon}$, where $\varphi_{M}$ are a complete orthonormal set of state-vectors, and $\Delta E_{M}$ is the difference between the energies of the state $M$ and the ground state. Here we neglect the positron contribution to the propagator. Using the electron propagator in the bound state, one readily obtains,

$$
\begin{aligned}
& \text { Fig. } 3(b)=\frac{\alpha}{(2 \pi)^{2}} \sum_{M}\left|\int d^{3} x \varphi_{0}^{\dagger}(x) \frac{\nabla}{m_{0}} \varphi_{M}(x)\right|^{2} \\
& \times \int d^{3} k\left[\frac{2}{3|\vec{k}|\left(\Delta E_{M}+|\vec{k}|\right)}-\frac{1-\vec{k}^{2} /\left(3\left(\vec{k}^{2}+\mu^{2}\right)\right)}{\sqrt{\vec{k}^{2}+\mu^{2}}\left(\Delta E_{M}+\sqrt{\vec{k}^{2}+\mu^{2}}\right)}\right]
\end{aligned}
$$

By using the identity $\sum_{M} \Delta E_{M}\left|\int d^{3} x \varphi_{0}^{\dagger}(x) \frac{\nabla}{m_{0}} \varphi_{M}(x)\right|^{2}=\frac{e^{2}}{2 m_{0}^{2}} \varphi_{0}^{\dagger}(0) \varphi_{0}(0)$ [37] and the completeness relation $\sum_{M} \varphi_{M}(x) \varphi_{M}^{\dagger}(y)=\delta^{3}(x-y)$, the energy shift for the ground state can be cast into a compact form,

$$
\begin{aligned}
\text { Fig. 3(b) - Fig. 3(a) } & =\frac{\alpha}{(2 \pi)^{2}} \sum_{M} \Delta E_{M}\left|\int d^{3} x \varphi_{0}^{\dagger}(x) \frac{\nabla}{m_{0}} \varphi_{M}(x)\right|^{2} \\
& \times \int d^{3} k\left[\frac{2}{3 \vec{k}^{2}\left(\Delta E_{M}+|\vec{k}|\right)}-\frac{1-\vec{k}^{2} /\left(3\left(\vec{k}^{2}+\mu^{2}\right)\right)}{\left(\vec{k}^{2}+\mu^{2}\right)\left(\Delta E_{M}+\sqrt{\vec{k}^{2}+\mu^{2}}\right)}\right] \\
& \approx \frac{4 \alpha_{\mathrm{em}}^{2}}{3 m^{2}} \varphi_{0}^{\dagger}(0) \varphi_{0}(0)\left[\ln \frac{\mu}{2 \Delta E}+\frac{5}{6}\right]
\end{aligned}
$$

where $\Delta E$ is a mean excitation energy. To arrive at the expression in the third line, we made use of a trick described in [36]. This result was first derived by Bethe in 1947 [37]. It is worthy to stress again that we computed the energy shift by evaluating the expectation value of the operator $m_{0} \bar{\Psi} \Psi$ rather than by calculating the self-energy correction dircetly. To justify the nonrelativistic treatment for electron, the scale $\mu$ should be chosen to be smaller than $m$, and was actually just set to be $m$ in the Bethe's original work, leading to a rather good agreement with the observed energy shift. To remove the $\mu$ dependence in a rigorous way, one has to include the high energy part [36], or in modern effective field language, performs a matching calculation for the vertex correction between the full QED and NRQED [33,34,38], which is however beyond the focus of this short note.

\section{CONCLUSION}

In summary, we have calculated the contribution from the trace anomaly of the QED energy momentum tensor to the hydrogen atom mass which is given in Eq. (10). It is shown to be related to the small part of the Lamb shift splitting that arises from the vacuum polarization effect in the perturbation theory up to the accuracy of the order $\alpha^{5}$. We stress again that this is the first attempt to understand the role of trace anomaly contribution to a bound state mass in a perturbation theory. We further computed the radiative corrections to the electron mass term of the QED EMT in the bound state, which turns out to be consistent with the electron self-energy calculation in the conventional treatment of the Lamb shift problem, as it should be.

The trace anomaly part is widely believed to be the dominant contribution to the mass of QCD bound states. It would be interesting to extend this analysis to heavy quarkonium system with an effective potential model. Actually, the perturbative calculation with the Cornell potential has achieved remarkable success in describing the spectrum of heavy quarkonium system. In analogy to hydrogen atom case, trace anomaly contribution to quarkonium mass is not a new source of bound state mass, and rather is part of already known potential energy. More precisely, trace anomaly contribution is related to the potential that violates conformal symmetry. The correction to the Coulomb part of the Cornell potential from the vacuum polarization effect can be computed in an analogous way as we did for hydrogen atom. The considerable amount of mass generated from the linear potential energy can also be attributed to trace anomaly contribution. However, the linear part of the Cornell potential inherently arises from the nonperturbative effect, and might be beyond the reach of the current method. Nevertheless, this certainly deserves further investigation. Moreover, the comparison between perturbative calculation and the Lattice result [39] will deepen our understanding of the role of trace anomaly contribution to a bound state in perturbation theory. 
To understand the trace anomaly contribution in the nonperturbative region, it might be necessary to invoke the Higgs-like mechanism induced by highly nontrivial QCD vacuum structure [40], and is beyond the power of the method presented in this short paper.

\section{ACKNOWLEDGMENTS}

J. Zhou thanks Yi-bo Yang and Jian-hua Gao for helpful discussions. Bao-dong Sun Thanks Ren-hong Fang and Rui Yu for helpful discussions. J. Zhou has been supported by the National Nature Science Foundations of China under Grant No. 11675093. B.-D. Sun has been supported by the National Natural Science Foundation of China under Grant No. 11947228 and the China Postdoctoral Science Foundation under Grant No. 2019M662316.

Note added.-After the manuscript was posted on arXiv, related preprints $[40,41]$ appeared soon, where the authors of $[40,41]$ claimed that their result differs from ours by a factor -2 .
[1] M. Brack, Phys. Rev. D 27, 1950 (1983).

[2] S. L. Adler, J. C. Collins, and A. Duncan, Phys. Rev. D 15, 1712 (1977).

[3] N. Nielsen, Nucl. Phys. B120, 212 (1977).

[4] J. C. Collins, A. Duncan, and S. D. Joglekar, Phys. Rev. D 16, 438 (1977).

[5] X.-D. Ji, Phys. Rev. Lett. 74, 1071 (1995).

[6] X.-D. Ji, Phys. Rev. D 52, 271 (1995).

[7] D. Kharzeev, Proc. Int. Sch. Phys. Fermi 130, 105 (1996).

[8] D. Kharzeev, H. Satz, A. Syamtomov, and G. Zinovev, Nucl. Phys. A661, 568 (1999).

[9] Y. Hatta and D.-L. Yang, Phys. Rev. D 98, 074003 (2018).

[10] Y. Hatta, A. Rajan, and D.-L. Yang, Phys. Rev. D 100, 014032 (2019).

[11] R. Wang, J. Evslin, and X. Chen, Eur. Phys. J. C 80, 507 (2020).

[12] F. Zeng, X.-Y. Wang, L. Zhang, Y.-P. Xie, R. Wang, and X. Chen, arXiv:2008.13439.

[13] R. Boussarie and Y. Hatta, Phys. Rev. D 101, 114004 (2020).

[14] P. Hagler, J. W. Negele, D. B. Renner, W. Schroers, T. Lippert, and K. Schilling (LHPC, SESAM Collaborations), Phys. Rev. D 68, 034505 (2003).

[15] M. Gockeler, R. Horsley, D. Pleiter, P. E. Rakow, A. Schafer, G. Schierholz, and W. Schroers (QCDSF Collaboration), Phys. Rev. Lett. 92, 042002 (2004).

[16] Y.-B. Yang, Y. Chen, T. Draper, M. Gong, K.-F. Liu, Z. Liu, and J.-P. Ma, Phys. Rev. D 91, 074516 (2015).

[17] Y.-B. Yang, J. Liang, Y.-J. Bi, Y. Chen, T. Draper, K.-F. Liu, and Z. Liu, Phys. Rev. Lett. 121, 212001 (2018).

[18] Y.-B. Yang, J. Liang, Z. Liu, and P. Sun (xQCD Collaboration), Proc. Sci., LATTICE2019 (2020) 001 [arXiv: 2003.12914].

[19] C. Lorce, Eur. Phys. J. C 78, 120 (2018).

[20] Y. Hatta, A. Rajan, and K. Tanaka, J. High Energy Phys. 12 (2018) 008.
[21] K. Tanaka, J. High Energy Phys. 01 (2019) 120.

[22] S. Rodini, A. Metz, and B. Pasquini, J. High Energy Phys. 09 (2020) 067.

[23] A. Metz, B. Pasquini, and S. Rodini, arXiv:2006.11171.

[24] M. V. Polyakov and P. Schweitzer, Int. J. Mod. Phys. A 33, 1830025 (2018).

[25] W. Cosyn, S. Cotogno, A. Freese, and C. Lorc, Eur. Phys. J. C 79, 476 (2019).

[26] M. V. Polyakov and B.-D. Sun, Phys. Rev. D 100, 036003 (2019).

[27] A. Freese and I. C. Clot, Phys. Rev. C 100, 015201 (2019).

[28] B.-D. Sun and Y.-B. Dong, Phys. Rev. D 101, 096008 (2020).

[29] S.-Z. Yang, J.-H. Gao, Z.-T. Liang, and Q. Wang, arXiv: 2003.04517.

[30] R. Tarrach, Nucl. Phys. B196, 45 (1982).

[31] S. L. Adler and W. A. Bardeen, Phys. Rev. D 4, 3045 (1971); 6, 734(E) (1972).

[32] V. Fock, Z. Phys. 63, 855 (1930).

[33] P. Labelle and S. Mohammad Zebarjad, Can. J. Phys. 77, 267 (1999).

[34] R. A. Porto, Phys. Rev. D 96, 024063 (2017).

[35] J.-W. Cui, Y.-L. Ma, and Y.-L. Wu, Phys. Rev. D 84, 025020 (2011).

[36] S. Weinberg, The Quantum Theory of Fields. Vol. 1: Foundations (Cambridge University Press, Cambridge, England, 2005), ISBN 978-0-521-67053-1, 978-0-51125204-4.

[37] H. Bethe, Phys. Rev. 72, 339 (1947).

[38] Y. Huang, Y. Jia, and R. Yu, arXiv:1901.04971.

[39] W. Sun, Y. Chen, P. Sun, and Y.-B. Yang ( $\chi$ QCD Collaboration), arXiv:2012.06228.

[40] X. Ji and Y. Liu, arXiv:2101.04483.

[41] X. Ji, Y. Liu, and A. Schäfer, arXiv:2105.03974. 